\title{
INTERACTION EFFECT OF BROODING RUMINATION AND INTEROCEPTIVE AWARENESS ON DEPRESSION AND ANXIETY SYMPTOMS
}

\author{
A thesis submitted \\ To Kent State University in partial \\ Fulfillment of the requirements for the \\ Degree of Master of Arts \\ by \\ Ryan Joseph Lackner \\ May 2016 \\ (C) Copyright \\ All rights reserved \\ Except for previously published materials
}


Thesis written by

Ryan Joseph Lackner

B.S., Indiana University, 2013

M.A., Kent State University, 2016

Approved by

$\overline{\text { David M. Fresco, Professor, Ph.D., Department }}$ of Psychological Sciences, Masters Advisor

Manfred van Dulmen, Professor, Ph.D., Interim Chair, Department of Psychological Sciences

James L. Blank, Ph.D., Dean, College of Arts and Sciences 


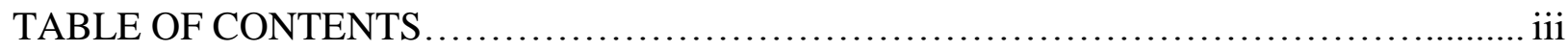

LIST OF TABLES ......................................................................... iv

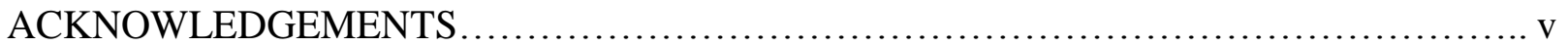

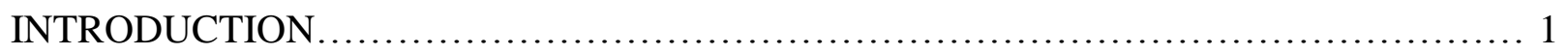

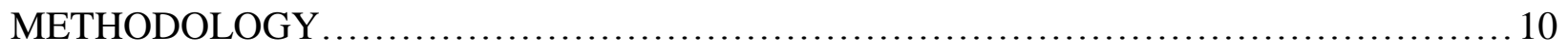

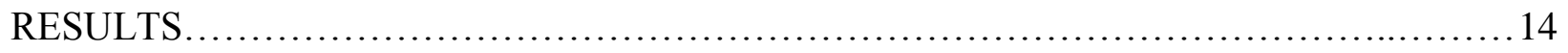

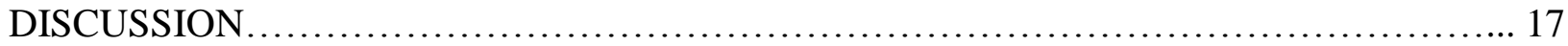

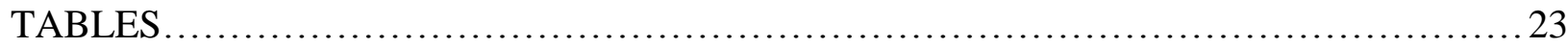

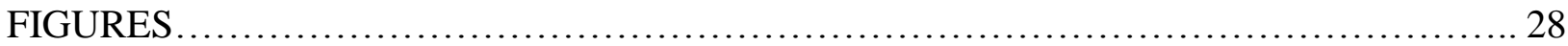

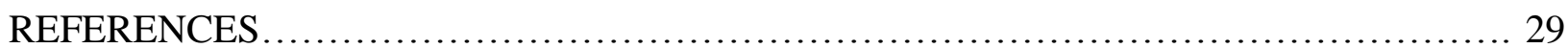




\section{LIST OF TABLES}

Table 1. Sample Descriptives and Correlations for Mood and Interoception Indicators.........23

Table 2. Intercorrelations among MASQ-S Dimensions................................ 24

Table 3. Linear Regression Model Predicting MASQ-S General Distress..................... 25

Table 4. Linear Regression Model Predicting MASQ-S Anxious Arousal..................... 26

Table 5. Linear Regression Model Predicting MASQ-S Anhedonic Depression................ 27 


\section{Acknowledgements}

I wish to thank my thesis committee for their time and helpful comments. I would especially like to thank Dr. David M. Fresco for his vital contribution toward my development as a critical thinker, researcher, and aspiring clinical psychologist. Finally, I would like to thank my father, Larry, and mother, Luann, for their perpetual love and support. 


\section{Introduction}

Two of the most commonly occurring emotional disorders, major depressive disorder (MDD) and generalized anxiety disorder (GAD), represent an enormous public health burden. Both disorders are similar in that impairment occurs within cognitive, affective, and somatic domains of functioning. Specifically, MDD is characterized by sustained depressed mood and/or loss of interest or pleasure in activities of daily living, and symptoms such as changes in appetite, sleep disturbance, changes in activity levels, loss of energy, feelings of guilt or worthlessness, difficulties concentrating, and suicidality (American Psychiatric Association, 2013). GAD is characterized by excessive worry that is difficult to control, restlessness or feeling keyed up or on edge, being easily fatigued, difficulty concentrating, irritability, muscle tension, and restless or unsatisfying sleep (American Psychiatric Association, 2013). Although significant progress in treating these disorders has been made, less than half of patients receiving a combination of medication and psychotherapy for MDD achieve remission (Casacalenda, Perry, \& Looper, 2002). Similarly, only 50-60\% of patients seeking treatment for GAD demonstrate clinically meaningful change (Borkovec \& Ruscio, 2001). Thus, further integrative research is needed to identify aberrant mechanisms that can be targeted for more efficient treatment interventions (Sanislow et al., 2010).

The capacity to reflect on one's self (i.e., self-referential processing) is a defining characteristic of human beings and may represent the default state of the brain (Raichle et al., 2001). The neuroanatomy of self-referential processing has been linked primarily to activity in the medial prefrontal cortex (mPFC; Buckner, Andrews-Hanna, \& Schacter, 2008), an area of the brain that is also implicated in the detection of emotionally salient stimuli (Morris et al., 1998; Phillips, Drevets, Rauch, \& Lane, 2003) as well as determining whether beliefs are "acceptable" or "unacceptable" (Paulus \& Stein, 2010). Activation in medial prefrontal regions has been 
observed in association with subjective reports and behavioral measures of mind wandering (Buckner et al., 2008; Christoff et al., 2009) which is akin to activity typically associated with the "default mode network" involved in self-regulation and monitoring of the internal milieu (Farb et al., 2007; Paulus \& Stein, 2010). Indeed, the mPFC is often found active during conditions in which attention is internally directed, processing self-relevant thoughts and beliefs (Paulus \& Stein, 2010). Thus, in the absence of task demands, neural activation tends to reflect an automatic tendency to engage in narrative, or evaluative cognitive processing (Mason et al., 2007; McKiernan, D'Angelo, Kaufman, \& Binder, 2006). In its healthy forms, self-referential processing is critical for self-regulation, adaptive social cognition, and planning of personally relevant goals (Mennin \& Fresco, 2013).

However, the human capacity for such higher-level self-consciousness may also be associated with dysfunction (Olatunji, Naragon-Gainey, \& Wolitsky-Taylor, 2013). Indeed, a shared aspect of both anxiety and depression is the altered experience of the individual with respect to self (Paulus \& Stein, 2010). Both affective psychopathologies exhibit the feature of negative self-referential processing (Northoff, 2007), though the content and temporal orientation of the negative thoughts may vary between depression and anxiety (Beckwé, Deroost, Koster, De Lissnyder, \& De Raedt, 2014). Negative self-referential thought in depression characteristically takes on the form of rumination, which describes a focus on negative past events along with a tendency to respond to sad mood by passively and repetitively focusing on the causes and consequences of negative emotions (Nolen-Hoeksema \& Morrow, 1993). Worry, a core feature of anxiety, focuses on possible negative events in the future and strategies to prevent such events from occurring (Borkovec \& Inz, 1990). Though distinct, recent literature suggests that rumination and worry are transdiagnostic processes that cut across MDD, GAD, and comorbid 
patients (Kircanski, Thompson, Sorenson, Sherdell, \& Gotlib, 2015). Thus, both rumination and worry represent dysfunctional forms of self-referential processing (Farb et al., 2015) and reflect cognitive processing that is temporally oriented toward the past or future - that is, away from present moment experience (Kircanski et al., 2015). Importantly, research has found that easily triggered self-evaluative processes to negative emotion may result in maladaptive cognitive reactivity, suggesting that the inability to disengage from such self-evaluative processes may ultimately be a hallmark of relapse risk (Farb, Anderson, Bloch, \& Segal, 2011). It is this endemic reliance upon networks supporting temporally extended processing that may obscure the recruitment of networks implicated in more immediate, perceptual self-reflection (Farb et al., 2007).

Another important aspect of self is corporeal awareness (Berlucchi \& Aglioti, 2009) or the "material self" (Craig, 2002). Research is beginning to uncover the importance of receiving, accessing, and appraising internal visceral signals, as such processing is critical for an organism's maintenance of desired physiological states, self-regulation, homeostasis, and in turn, survival (Craig, 2013; Farb et al., 2015; Paulus, 2007). The term “interoception” has been variously employed to reference the awareness of body signals (Mussgay, Klinkenberg, \& Rüddel, 1999), physiological feedback from the whole body (Wiens, 2005), and afferent information that arises from anywhere and everywhere within the body (Cameron, 2001). Neuroanatomical evidence supports such broad definitions as research has indicated that a class of afferent fibers that monitor the physiological state of all internal organs of the body converge in the insular cortex (Craig, 2002; Paulus \& Stein, 2006). The insula, considered the interoceptive center of the brain (Critchley, Wiens, Rotshtein, Öhman, \& Dolan, 2004), is critical for evaluating the potential impact of stimuli on the body (Paulus \& Stein, 2006), including 
generation and regulation of affective responses and detection of emotionally salient stimuli (Paulus \& Stein, 2010).

The notion that visceral signal perception is an essential component of emotional experience is shared by numerous psychophysiological, or "peripheral feedback" theories of emotion (Pollatos, Kirsch, \& Schandry, 2005). James (1884) postulated "that bodily changes follow directly the perception of the exciting fact, and that our feeling of the same changes as they occur IS the emotion". A century later, Schachter and Singer (1962) maintained that visceral arousal is necessary for the occurrence of emotions, but emphasized that the unique valence of an emotional state is defined by cognitive labeling and that arousal determines the intensity of the emotional experience. More recently, Damasio $(1994,1999)$ proposed the "somatic marker hypothesis," a theory suggesting that body signals, or "somatic markers," are represented and regulated in emotion circuitry in the brain, which impact decision-making in uncertain situations. Although empirical evidence for these theories is mixed (Dunn, Dalgleish, \& Lawrence, 2006; Prinz, 2004), it remains highly plausible that visceral signal perception is intimately bound with emotional experience.

Various techniques have been developed to quantify interoceptive awareness in experimental settings. Efforts have focused on cardiovascular perception primarily because heartbeats are distinct autonomic events that are easily measured, non-invasive, and clearly associated with emotion (Barrett et al., 2004; Herbert, Pollatos, Flor, Enck, \& Schandry, 2010; Wiens, Mezzacappa, \& Katkin, 2000). The most widely used methods are heartbeat tracking (Ehlers \& Breuer, 1992; Schandry, 1981) and heartbeat discrimination tasks (Whitehead, Drescher, Heiman, \& Blackwell, 1977; Yates, Jones, Marie, \& Hogben, 1985). Typically, heartbeat tracking tasks require participants to silently count their heartbeats during various, 
intervals of time. Performance is captured by an error score, which reflects the difference between the number of heartbeats reported by the individual compared to the actual number of heartbeats that occurred in a given time period as recorded by physiological monitoring equipment. Heartbeat discrimination tasks require participants to determine whether their heartbeats occur simultaneously with trains of external stimuli (e.g. lights, tones), which are presented at various time delays in relation to an individual's onset of R-wave of an electrocardiogram. "Good perceivers" on these tasks are individuals whose subjective reports align with their objective number of recorded heartbeats.

Performance on heartbeat perception tasks is widely distributed throughout normative populations (Schandry, 1981; Whitehead et al., 1977). Studies have generally found a positive correlation between visceral signal sensitivity and perceived intensity of emotional experience (Critchley et al., 2004; Ferguson \& Katkin, 1996; Herbert et al., 2010; Montoya, Schandry, \& Müller, 1993; Schandry, 1981; Wiens et al., 2000); however, results are not equivocal (see Barrett et al., 2004). Other studies examining the relationship between visceral signal perception and levels of arousal in emotionally stimulating contexts have found that the two appear unrelated (Herbert et al., 2010; Wiens et al., 2000), suggesting that it is not the mere occurrence of peripheral changes in the body, but their perception that affects the intensity of emotional experience and that visceral self-perception is independent of sympathetic arousal. In general, men have been documented as scoring relatively higher than women on perception accuracy; however the results are less consistent after controlling for percentage of body fat (e.g. lean men vs. women; Cameron, 2001). Other potentially confounding factors include the effects of blood systolic blood pressure (O'Brien, Reid, \& Jones, 1998), individual fitness level (Cameron, 2001), 
heart-rate and heart-rate variability (Knapp-Kline \& Kline, 2005), and overall cardiodynamics (Schandry, Bestler, \& Montoya, 1993).

A growing body of research has shown that interoceptive awareness is implicated in decision making (Dunn et al. 2010a; Dunn, Evans, Makarova, White, \& Clark, 2012; Kirk, Downwar, \& Montague, 2011; Werner, Jung, Duschek, \& Schandry, 2009), affect regulation (Füstös, Gramann, Herbert, \& Pollatos, 2012; Sze, Gyurak, Yuan, \& Levenson, 2010), and in other domains of cognitive and behavioral functioning (Herbert et al., 2010; Pollatos \& Schandry, 2008; Pollatos, Schandry, Auer, \& Kaufmann, 2007; Werner, Mannhart, Reyes Del Paso, \& Duschek, 2014; Werner, Peres, Duschek, \& Schandry, 2010). Specifically, Kirk and colleagues (2011) found that interoception drives increased rational decision-making while playing the ultimatum game, a two-person monetary exchange task. Füstös, Gramann, Herbert, and Pollatos (2012) provided electrophysiological evidence for the relevance of interoceptive awareness in emotion regulation; specifically, interoceptive awareness facilitated downregulation of affect-related arousal during a cognitive reappraisal task, suggesting that the more aware a person is of ongoing bodily processes, the more successful this person will be in regulating emotions in response to negative affect. Another study found that individuals who demonstrated superior interoceptive awareness revealed greater arousal (i.e. greater P300 amplitudes) in response to a set of emotional stimuli (Pollatos et al., 2005). Finally, previous work has also found that individuals who show greater interoceptive sensitivity spend less physical effort due to their better perception of bodily feedback coming from their cardiac system (Herbert, Ulbrich, \& Schandry, 2007).

Previous research also suggests that the focus of attention on directly experienced sensations (i.e., visceral signals) may represent a critical aspect of well-being (Davidson, 2004; 
Watkins \& Moulds, 2005). Evidence has shown that localized attention to body sensations enables subsequent gains in emotional and cognitive regulation by enhancing sensory information processing in the brain (Kerr, Sacchet, Lazar, Moore, \& Jones, 2013). The notion that more experiential forms of attention are important for well-being is shared by previous theories which distinguish maladaptive verses adaptive modes of mind, or self-focus (Teasdale, 1999; Watkins \& Teasdale, 2004). For example, Interacting Cognitive Subsystems (ICS) theory (Teasdale, 1999) proposes that direct experience of one's sensations (i.e., mindful experiencingbeing mode) allows the integration of multiple elements of experience, which subsequently facilitates emotional well-being. Similarly, experiential forms of self-awareness appear to be more adaptive than analytical forms of self-reference in recovered depressed patients (Watkins \& Teasdale, 2004). From this reasoning, attention to body signals in the present moment may disrupt forms of self-reference that underlie maladaptive cognitive reactivity (Farb et al., 2011). That is, developing a heightened sense of interoception may have its positive effect in disengaging habitual, evaluative cognitive processes such as rumination and worry that shift attention toward the past or future (Killingsworth \& Gilbert, 2010), and in turn, contribute to affective disorder onset and relapse (Farb et al., 2011).

However, greater interoceptive ability may also contribute to anxiety and panic related disorders when catastrophized (Farb et al., 2015). Although the exact relationship between interoceptive awareness and psychopathology remains unclear, the literature generally supports a positive association between cardiac awareness and anxiety (Domschke, Stevens, Pfleiderer, \& Gerlach, 2010; Ehlers \& Breuer, 1992; Paulus \& Stein, 2010; Pollatos, Traut-Mattausch, \& Schandry, 2009; Schandry, 1981). For example, one study found that patients with panic disorder, infrequent panickers, and individuals with other anxiety disorders reported greater 
cardiac awareness compared to healthy controls (Ehlers \& Breuer, 1992). In a similar study with children, increased panic symptoms were associated with enhanced heartbeat perception (Eley, Stirling, Ehlers, Gregory, \& Clark, 2004). Increased cardiac awareness has also been found in social phobia (Pineles \& Mineka, 2005). Such findings have engendered the hypothesis that enhanced body awareness may be implicated in the etiology and maintenance of anxiety disorders (Mehling et al., 2012), though recent evidence suggests that hypervigilance to body symptoms is not necessarily a bottom-up dispositional tendency, but rather a metacognitive process related to threatening beliefs about somatic sensations (Yoris et al., 2015). Relatedly, it has been proposed that anxiety sensitivity (Reiss, Peterson, Gursky, \& McNally, 1986), or the tendency to view body signals as threatening, is mediated through processes underlying the perception of heightened interoceptive prediction signals (Paulus \& Stein, 2006); that is, anxietyprone individuals may not have a heightened baseline interoceptive state, but an exaggerated expectation (i.e., belief) about future body states. Thus, whether greater interoceptive ability contributes to or detracts from well-being may depend on how such awareness is understood, and how to skillfully relate to body sensations remains a question for further inquiry (Farb et al., 2015).

Conversely, most studies have found that heartbeat perception is less accurate in depressed individuals (Dunn, Dalgleish, Ogilvie, \& Lawrence, 2007; Paulus \& Stein, 2010); however, Dunn and colleagues (2007) noted that an increase from low to moderate depression severity resulted in less accurate heartbeat perception, whereas an increase from moderate to severe depression resulted in increased perception accuracy. Overall, there seems to be an interactive effect of depressive and anxious symptomatology on interoceptive sensitivity. Dunn and colleagues (2010b) examined the interaction of scores on depression-specific anhedonia and 
anxiety-specific arousal symptom dimensions on interoceptive accuracy and found that as anhedonia symptoms increased, the relationship between arousal and interoceptive accuracy became less marked. Pollatos and colleagues (2009) also demonstrated a moderating effect of anxiety on the relationship of interoception and depression. Finally, Furman et al. (2013) reported poorer heartbeat perception accuracy in a sample of depressed females without anxiety comorbidities. In summary, accumulating evidence suggests abnormal somatic signaling and interoceptive dysfunction in depression (Harshaw, 2014).

A recent neuroanatomical and processing model (Paulus \& Stein, 2010) for depression and anxiety incorporates the connections between increased but low fidelity afferent interoceptive input with self-referential and belief-based states. Specifically, Paulus and Stein (2010) propose that propositional states that refer to the state of the individual, which are processed in the $\mathrm{mPFC}$, contribute significantly to the evaluation of anticipatory interoceptive signals; it is assumed that an exaggeration of the valence (i.e., positive or negative) relative to the individual's internal state plays a role in enhancing the aversive aspects of predictive body signals associated with potential negative outcomes. Furthermore, Paulus and Stein (2010) propose that individuals at risk for anxiety and depression exhibit a reduced signal to noise ratio of interoceptive afferents; for example, a heartbeat or sensation related to breathing interpreted with a negative valance, or belief-based process (e.g., "there is something wrong with my heart") will increase the sympathetic nervous system response and result in withdrawal or avoidance behaviors. Consequently, low fidelity interoceptive afferents result in overactive top-down brain modulatory areas (e.g., mPFC) that engage constantly to differentially amplify or attenuate body signals. In summation, the model proposed by Paulus and Stein (2010) proposes that a change in one's internal state, due either to an increased attentional bias toward negative self-view 
(depression) or threat (anxiety), generates beliefs that are implicated in the interpretation of afferent internal body signals.

Thus, in order to better understand the dysfunctions observed in depression and anxiety with respect to self, the current study sought to examine the integration of interoceptive processes with self-referential processes in the context of depression and anxiety symptoms. In particular, the aim of the current study was to further elucidate the relationship between negative self-referential processing (i.e., rumination), interoceptive awareness, and depression and anxiety symptoms. Although previous studies have separately examined rumination and interoceptive awareness in the context psychopathology, research has yet to investigate the integration of brooding rumination and interoceptive awareness in the context of affective psychopathology. We hypothesized an interaction effect of brooding rumination and interoceptive awareness on depression and anxiety-related symptoms. Specifically, we hypothesized that high levels of rumination coupled with low levels of interoceptive awareness would be associated with the highest levels of depression and anxiety symptoms, and that lower levels of rumination coupled with higher levels of interoceptive awareness would be associated with lower levels of depression and anxiety symptoms.

\section{Methodology}

Participants

Data were obtained from eighty-two college students at a large midwestern university. Of these participants, 67 were female $(77.3 \%)$ and the mean age was 19.63 years $(S D=2.04)$. The sample consisted of Caucasian (69.3\%), African American (12.5\%), Biracial (4.5\%), Latino 
(3.4\%), Asian (2.3\%), and Other (6.8\%) participants. All participants provided written informed consent. A power analysis determined the sample size necessary to reveal a medium effect size. Medication use in the current sample included two participants taking benzodiazepines, seven participants taking anti-depressants, and four participants taking ADHD medication. Seven participants self-reported a current anxiety diagnosis. Two participants self-reported a current depression diagnosis, and two participants self-reported comorbid anxiety and depression diagnoses. One participant reported diabetes, and one participant reported high blood pressure.

\section{Procedure}

Following consenting procedures, participants' weight, height, and blood pressure were measured. Participants then completed a series of questionnaires (described below), in addition to other measures not included in the present analysis.

Participants were fitted with non-polarizable $\mathrm{Ag}-\mathrm{AgCl}$ adhesive disposable electrodes (Biopac Systems Inc., Santa Barbara, California, USA) attached to the right mid-clavicle and lower left rib cage for electrocardiogram (ECG) measurement. Two additional non-polarizable $\mathrm{Ag}-\mathrm{AgCl}$ electrodes positioned on the medial phalanx of the index and middle fingers recorded skin conductance. Participants were instructed to remain as still as possible during recording to minimize the occurrence of motion artifacts. Baseline measures of ECG and skin conductance were collected for 3 minutes prior to experimental task initiation. Participants then completed the heartbeat perception task. ECG was recorded using a BIOPAC MP150 unit (Biopac Systems Inc., Santa Barbara, California, USA) acquiring data at $250 \mathrm{~Hz}$, connected to a PC running Acqknowledge software. A live-feed camera during experimental procedures and a postquestionnaire following procedures ensured participant compliance. 


\section{Interoceptive Awareness}

Interoceptive awareness was assessed using a heartbeat perception task similar to the one developed by Schandry (1981), which has been used widely throughout the literature (e.g., Herbert et al., 2010; Herbert et al., 2007; Pollatos et al., 2007). Participants were instructed to silently count the number of heartbeats they perceived during various intervals of time $(25,35$, and 55 seconds), each repeated twice for a total of 6 trials, with a 30 second rest period given between each trial. A 12 second practice trial preceded experimental trials. Participants were instructed to "concentrate on heart activity without feeling for a pulse or altering the breath" and to report the number of heartbeats they perceived for each trial. Each trial began with an $800 \mathrm{~Hz}$, $100 \mathrm{~ms}$ tone, indicating that the participant should be prepared to start perceiving their heart activity. A $1000 \mathrm{~Hz}, 50 \mathrm{~ms}$ start tone was presented three seconds later, and the same auditory cue signified the end of each trial. Participants were not informed about the length of each interval, nor about their performance accuracy. Participants also completed 3 trials of time perception to determine whether performance on the heartbeat tracking task was attributable to ability to estimate time (Dunn et al., 2006). Lengths of the 3 time intervals were 23, 40, and 56 seconds. Participants were also asked to provide an estimate of their resting heart rate.

Heartbeat perception scores were calculated by taking the absolute value of the recorded heartbeats minus the estimated heartbeats, divided by the recorded number of heartbeats, and then multiplying by 100 to express this as a percentage, for each trial (ABS[actual - estimated] $\div$ actual] x 100). Performance was averaged across all six trials and this average was subtracted from 100 to express that the higher the perception score, the better the accuracy. 
Previous studies have selected a cut-off score of 0.85 to distinguish between poor (below 0.85) and good (above 0.85) perceivers (Herbert et al., 2007; Montoya et al., 1993; Pollatos et al., 2005; Pollatos et al., 2007), which has been shown to be appropriate for distinguishing between individuals who substantially differ in cardiac awareness. However, there is some controversy about whether heartbeat perception scores should be analyzed as a continuous (mean error score) or categorical (good vs. poor) variable (Ehlers, 1998); specifically, it has been argued that dichotomous scoring is appropriate only when the results obtained from the heartbeat perception task conform to a bimodal distribution, or when participates perform poorly. Given that performance was adequate and that IA scores conformed to a normal distribution in the current study, interoceptive awareness accuracy was treated as a continuous variable.

\section{Rumination}

Rumination was measured using the Ruminative Response Scale (RRS). The RSS a 22 item subscale of the Response Styles Questionnaire (RSQ; Nolen-Hoeksema \& Morrow, 1991) that assesses people's tendencies to ruminate when distressed. Participants rate their agreement with statements on a Likert-type scale ranging from 1 (almost never) to 4 (almost always). The items describe responses to depressed mood that are self-focused, symptom-focused, and focused on the possible consequences and causes of their mood. The RSS features two subscales that capture distinguishable components of rumination: reflective pondering and brooding. Notably, the brooding scale is less confounded with depression and more useful in gaining a finer understanding of the relationship between rumination and depression (Armey et al., 2009; Treynor, Gonzalez, \& Nolen-Hoeksema, 2003). The RSS has demonstrated high internal consistency (Cronbach's alpha .90) as well as acceptable convergent and predictive validity 
(Butler \& Nolen-Hoeksema, 1994; Nolen-Hoeksema \& Morrow, 1991). RSS total score reliability for the current study was .92 and brooding subscale reliability was .77.

\section{Depression and Anxiety-related Symptomatology}

Depression and anxiety symptoms were assessed using the short form of the Mood and Anxiety Symptom Questionnaire (MASQ-S; Watson \& Clark, 1991). The MASQ-S is a 62 item self-report questionnaire that measures depression-specific anhedonia symptoms (22 items), anxiety-specific hyper-arousal symptoms (17 items), and general distress symptoms common to anxiety and depression (23 items). Participants indicate on a 5 point scale, ranging from 1 (not at all) to 5 (extremely), how much they have experienced each symptom during the past week as well as the day of testing. The MASQ-S demonstrated good convergent and discriminant validity, reliability, and a stable factor structure in student, community, and adult substance abuse patient samples (Watson et al., 1995a, 1995b). MASQ-S total score reliability for the current study was .89 . Reliability was also calculated for each subscale (general distress: $=.92$; anxious arousal $=.77$; anhedonic depression $=.76$ ).

\section{Results}

Scores obtained from the MASQ-S dimensions indicated that the current sample approaches levels of anxiety previously found in clinically diagnosed groups (Pizzagalli, Iosifescu, Hallett, Ratner, \& Fava, 2008; Watson et al., 1995a) and scores on the anhedonia and general distress dimensions were similar to scores obtained by Dunn and colleagues (2010b).

Overall, interoceptive awareness evidenced a negative association with self-reported depression and anxiety symptoms. Specifically, correlational analyses revealed significant associations 
between heartbeat perception task performance and MASQ-S dimensions of anhedonic depression, anxious arousal, general distress symptoms. Interoceptive awareness was not significantly associated with rumination. Table 1 summarizes descriptive statistics and correlations for mood and interoception measures. Table 2 reports intercorrelations among MASQ-S dimensions.

We examined the extent to which interoceptive awareness was related to potential confound variables such as body mass index (BMI), blood pressure, skin conductance, heart rate variability, exercise, resting heart rate, estimates about resting heart rate, current depression or anxiety diagnosis, prescribed psychotropic drug use, and ability to estimate time. Interoceptive awareness was not significantly associated with BMI, blood pressure, skin conductance, HRV, minutes of exercise per week, depression or anxiety diagnoses, or prescribed psychotropic drug use. Slower resting heart rate, estimates about resting heart rate, and time accuracy were all significantly associated with interoceptive awareness (see Table 1). As such, we conducted an additional regression analysis with estimate of resting heart rate and time estimation accuracy as nuisance covariates. After controlling for these factors, the regression results remained consistent as described below.

We tested a regression model that examined interoceptive awareness, brooding rumination, and their interaction as predictors of self-reported depression and anxiety symptoms for each MASQ-S dimension. Two outliers were removed (Z-scores > 3 SDs from mean), two participants had missing data, and fifteen participants' data from the heartbeat perception task were removed due to unusable ECG data (e.g., noise artifacts, non-compliance). Greater interoceptive awareness was associated with lower scores on the general distress dimension, and greater brooding rumination was associated with higher scores on the general distress dimension. 
For the general distress dimension, adding the interaction of interoceptive awareness and brooding rumination significantly improved model fit. Tables 3 describes the regression coefficients and model associated with the general distress dimension. Interoceptive awareness was not associated with anxious arousal; however, greater interoceptive awareness was associated with lower scores on the dimension of anhedonic depression. Greater brooding rumination was associated with higher scores on the general distress dimension. The addition of the interaction of interoceptive awareness and brooding rumination did not improve the fit of either model predicting anxious arousal or anhedonic depression, though the interaction term evidenced an association at the level of a non-significant trend for the model predicting anxious arousal. Tables 4 and 5 describe the regression coefficients and model associated with anxious arousal and anhedonic depression, respectively.

To further examine the form of the significant interaction in the model for general distress, we conducted simple slope analyses for the association between interoceptive awareness and general distress at low (-1 SD below the mean), moderate (mean), and high (+1 SD above the mean) levels of brooding rumination. Notably, high levels of brooding rumination coupled with low levels of interoceptive awareness were associated with the highest levels of depression and anxiety-related distress, whereas lower levels of brooding rumination coupled with higher levels of interoceptive awareness were associated with lower levels of depression and anxietyrelated distress. The simple slopes tests revealed a significant negative association between interoceptive awareness and general distress, and the strength of this association was more strongly related to general distress for high levels of brooding rumination, $\beta=-.41, t(77)=-4.37$, $p<.001$, than for moderate levels of brooding rumination $\beta=-.19, t(77)=-2.96, p<.01$. At low 
levels of brooding rumination, the slope was not significantly different from zero, $\beta=.01, t(77)$ $=.10, p=.92$. The interaction is illustrated in Figure 1 .

\section{Discussion}

The present study examined the relationship between negative self-referential processing (i.e., rumination), interoceptive awareness, and depression and anxiety symptoms. We hypothesized brooding rumination would moderate the relationship of interoceptive awareness to depression and anxiety-related symptoms such that high levels of brooding rumination coupled with low levels of interoceptive awareness would be associated with the highest levels of depression and anxiety, and that lower levels of rumination would be associated with a less marked effect. The hypothesis was supported for the statistical model predicting MASQ-S general distress. The results did not support an interaction effect of brooding rumination and interoceptive awareness on symptom dimensions of anxious arousal or anhedonic depression; however, greater interoceptive awareness was associated with lower scores on the dimension of anhedonic depression and greater brooding rumination was associated with higher scores on both the anxious arousal and anhedonic depression dimensions.

The findings provide further support for the conceptualization of anxiety and depression as disorders involving an integration of interoceptive processes with self-referential processes (Paulus \& Stein, 2010). In corroboration with previous work (Nolen-Hoeksema \& Morrow, 1993), results indicated that rumination was positively associated with self-reported depression and anxiety symptoms, whereas increased interoceptive awareness evidenced a negative association with depression and anxiety symptoms. The finding that interoceptive awareness was inversely related to anhedonia symptoms replicates previous work (Dunn et al., 2010b; 
Furman et al., 2013). The inverse association between interoceptive awareness and anxious arousal is contrary to results obtained in previous studies that evidence a positive association between interoception and anxiety (e.g., Domschke et al., 2010; Ehlers \& Breuer; Pollatos et al., 2009). One explanation for this is that arousal may only predict greater interoception in individuals who are not anhedonic (Dunn et al., 2010b). Given recent work demonstrating the significance of metacognitive interoception in the interpretation of body signals (Yoris et al., 2015), it is also possible that, overall, the current sample did not view their body signals as threatening, although future work should aim to measure metacognitive interoception in addition to interoceptive awareness.

One possible explanation for the interaction finding is that individuals with low fidelity interoceptive afferents recruit overactive top-down modulatory areas (e.g., mPFC) that underlie negative self-referential processing; individuals reporting the highest levels of anxiety and depression symptoms may exhibit a reduced signal to noise ratio of interoceptive afferents (Paulus \& Stein, 2010). However, given the correlational nature of the present study, this explanation remains speculative. In future research, it will be important to investigate this effect using fMRI to test this hypothesis. Current fMRI evidence supports two distinct, but habitually integrated modes of self-reference: extended self-reference (i.e., narrative focus; NF), which refers to the self as experienced across time, and momentary self-reference (i.e., experiential focus; EF) centered on present moment experience (Farb et al., 2007). Functional connectivity analyses conducted by Farb and colleagues (2007) demonstrated a strong coupling between the right insula (associated with $\mathrm{EF}$ ) and the $\mathrm{mPFC}$ (associated with NF) that became uncoupled as a result of attention training (i.e., mindfulness meditation). Other research has also documented afferent and efferent connections between the insula and mPFC (Paulus \& Stein, 2010). As 
suggested by Farb et al. (2007), the development of moment-to-moment self-reference (including interoceptive awareness) may facilitate a shift away from neural processes devoted to more abstract, narrative forms of self- reference. Whether this is attributable to an increase of the signal to noise ratio of interoceptive afferents and a decrease of overactive top-own brain modulatory areas (Paulus \& Stein, 2010) remains unclear. Future fMRI research may also want to consider whether there is an optimal signal to noise ratio of interoceptive afferents.

Awareness of the breath or body sensations as a method of focusing attention in the present moment is emphasized in many mindfulness-based interventions. In fact, it has been proposed that the development of interoceptive awareness and focal attention to bodily sensations in early mindfulness training is one of the primary ways in which such practices promote cognitive change (Farb et al., 2015; Kerr et al., 2013) via the reduction of evaluative self-referential processing and enhancement of present-moment experience (Tang, Hölzel, \& Posner, 2015). Accordingly, many imaging studies have shown IA-specific functional plasticity in insular regions (Craig, 2009; Farb, Segal, \& Anderson, 2013; Farb et al., 2007), suggesting the possibility that enhanced insula activity reflects an amplified awareness of present-moment experience (Tang et al., 2015). A possible mechanism by which interoceptive awareness may serve to mitigate the association between affective symptoms and rumination is via the deliberate switching of attention away from ruminative tendencies and toward body signals. This hypothesis follows directly from theories proposing that experiental forms of self-awareness appear to be more adaptive than analytical forms of self-reference (Teasdale, 1999; Watkins \& Teasdale, 2004).

Although heartbeat perception tasks are generally well validated and reliable (Cronbach's a .69-90; Dunn et al., 2007), questions remain as to whether these tasks represent the "gold 
standards" of interoceptive awareness measurement (Knapp-Kline \& Kline, 2005; Knoll \& Hodapp, 1992; Ring, Brener, Knapp, \& Mailloux, 2015). For example, in a recent study conducted by Ring and colleagues (2015), findings demonstrated that significant increases in the accuracy of heartbeat counting tasks were mediated by non-sensory processes rather than by training participants to detect heartbeat sensations; specifically, performance improved solely on the basis of beliefs about heart rate, leading the authors to conclude that data generated by heartbeat counting tasks are uninterpretable without additional control measures such as beliefs about heart rate. Although the current findings demonstrated a correlation between performance on the heartbeat perception task and estimates of resting heart rate, results remained consistent after controlling for such factors.

Nonetheless, future research may want focus on the development of interoceptive awareness tasks that are less confounded by non-sensory processes. One study found that performance on a heartbeat tracking task correlated with sensitivity for gastric signals using a water load test, providing evidence for interoceptive coherence across visceral modalities (Herbert, Muth, Pollatos, \& Herbert, 2012). Other investigators have operationalized interoceptive awareness in terms of respiratory sensitivity or accuracy, typically using various forms of the respiratory load task (Zechman, Hall, \& Hull, 1957). These tasks require participants to detect when resistance has been introduced into a tube through which they are breathing (Davenport, Chan, Zhang, \& Chou, 2007; Zhao, Martin, \& Davenport, 2002) or to discriminate among various resistive loads introduced into the airway (Webster \& Colrain, 2000). Recent neuroanatomical and neurophysiological evidence suggests a correlation between heartbeat perception and respiratory sensitivity. Research has indicated that a class of afferent fibers that monitor the physiological state of all internal organs of the body converge in the 
insular cortex (Craig, 2002; Paulus \& Stein, 2006). Indeed, evidence from other studies has demonstrated activation in the anterior insular cortex during heartbeat perception (Critchley et al., 2004), bladder and stomach distension, sexual arousal, sensual touch, and pain (Craig, 2009). Findings by Herbert and colleagues (2012) also suggest that interoceptive ability extends across visceral modalities. Taken together, a battery of tasks may represent a more reliable measurement of interoceptive awareness, as opposed to reliance on a single, potentially confounded heartbeat perception paradigm.

Other limitations of the current study should be noted. First, the sample was comprised of a convenient population of primarily female, Caucasian, college students. Although the current sample evidenced levels of anxiety commonly found in clinically diagnosed groups (Pizzagalli et al., 2008; Watson et al., 1995b), an additional limitation is potential range restriction effects given the non-clinical sample. Thus, future work should seek to replicate the findings in more diverse populations, before strong conclusions are drawn. Second, only one aspect of interoception was examined in the current study, as we operationalized interoceptive awareness as accuracy on a heartbeat perception paradigm. Future work should test this effect among other forms of interoception (e.g., interoceptive sensitivity, interoceptive coherence, metacognitive interoception; Farb et al., 2015). In future research, it will also be important to utilize more behavioral-based measures of rumination, given the limitations of self-report measures.

In summary, the present study evidenced an interaction effect of brooding rumination and interoceptive awareness on depression and anxiety-related symptomatology, such that high levels of brooding rumination coupled with low levels of interoceptive awareness were associated with the highest levels of symptoms of depression and anxiety. The findings provide further support 
for the conceptualization of anxiety and depression as disorders involving an interaction between interoceptive processes and negative self-referential processes. 
Table 1. Sample Descriptives and Correlations for Mood and Interoception Indicators.

\begin{tabular}{lrrr}
\hline & \multicolumn{3}{c}{ Correlation with } \\
Indicator & Mean & \multicolumn{1}{c}{ SD } & IA \\
\hline Interoception \% Accuracy & 63.04 & 17.28 & - \\
MASQ-S - total score & 126.42 & 29.9 & $-0.38^{* *}$ \\
MASQ-S - general distress depression & 23.78 & 9.11 & $-0.36^{* *}$ \\
MASQ-S - general distress anxiety & 21.14 & 6.58 & $-0.35^{* *}$ \\
MASQ-S - anxious arousal & 25.73 & 7.56 & $-0.23^{*}$ \\
MASQ-S - anhedonia & 55.77 & 13.32 & $-0.32^{* *}$ \\
RSQ-RSS - brooding & 10.03 & 3.55 & -0.18 \\
RSQ-RSS - pondering & 8.97 & 3.34 & -0.08 \\
Time \% Accuracy & 61.57 & 9.34 & $-0.42^{* *}$ \\
Body Mass Index & 26.70 & 6.16 & -0.08 \\
Resting Heart Rate & 75.39 & 10.13 & $-0.25^{*}$ \\
Resting HR Belief \% Accuracy & 56.78 & 24.01 & $0.31^{* *}$ \\
Heart Rate Variability (SDNN) & 69.66 & 27.54 & 0.18 \\
Physical Activity (min/week) & 244.18 & 181.52 & 0.19 \\
Blood Pressure (S/D) & 1.73 & 0.23 & -0.07 \\
Skin conductance (spontaneous fluctuations/min) & 3.38 & 4.04 & 0.04 \\
\hline
\end{tabular}

Note: MASQ-S = Mood and Anxiety Symptom Questionnaire - Short Form. RSQ-RSS = Response Styles Questionnaire - Ruminative Response Scale. * Correlation is significant at the 0.05 level (2-tailed). ** Correlation is significant at the 0.01 level (2-tailed). 
Table 2. Intercorrelations among MASQ-S Dimensions.

\begin{tabular}{lccc}
\hline & 1 & 2 & 3 \\
\hline MASQ-S - general distress (1) & - & & \\
MASQ-S - anxious arousal (2) & $.70^{* *}$ & - & \\
MASQ-S - anhedonia (3) & $.60^{* *}$ & $.44^{* *}$ & - \\
\hline
\end{tabular}

Note: MASQ-S = Mood and Anxiety Symptom Questionnaire - Short Form. ** Correlation is significant at the 0.01 level (2-tailed). 
Table 3. Linear Regression Model Predicting MASQ-S General Distress.

\begin{tabular}{|c|c|c|c|c|c|c|c|c|c|c|}
\hline \multirow[b]{2}{*}{ Step } & \multirow[b]{2}{*}{ Predictor } & \multicolumn{4}{|c|}{ Regression Coefficients } & \multicolumn{5}{|c|}{ Regression Model } \\
\hline & & B & $p r$ & $t$ & $p$ & $R^{2}$ & $\begin{array}{c}F \\
\text { change }\end{array}$ & $d f$ & $p$ & $\begin{array}{c}\text { Cohen's } \\
f^{2} \\
\end{array}$ \\
\hline 1 & IA & -0.25 & -0.35 & -3.30 & .001 & & & & & \\
\hline & $\begin{array}{l}\text { Brooding } \\
\text { Rumination }\end{array}$ & 2.09 & 0.54 & 5.69 & .000 & 0.38 & 25.77 & 2,79 & .000 & 0.65 \\
\hline 2 & $\begin{array}{l}\text { IA } x \\
\text { Brooding } \\
\text { Rumination }\end{array}$ & -0.06 & -0.31 & -2.82 & .006 & 0.43 & 7.98 & 1,78 & .006 & 0.1 \\
\hline
\end{tabular}

Note. Both Step 1 predictors are centered; $B=$ unstandardized regression coefficient; $p r=$ partial correlation between predictor and dependent measure; $R^{2}=$ Variance explained by the model (e.g., . $40=40 \%$ ); IA = Interoceptive Awareness 
Table 4. Linear Regression Model Predicting MASQ-S Anxious Arousal.

\begin{tabular}{|c|c|c|c|c|c|c|c|c|c|c|}
\hline \multirow[b]{2}{*}{ Step } & \multirow[b]{2}{*}{ Predictor } & \multicolumn{4}{|c|}{ Regression Coefficients } & \multicolumn{5}{|c|}{ Regression Model } \\
\hline & & B & $p r$ & $t$ & $p$ & $R^{2}$ & $\begin{array}{c}F \\
\text { change }\end{array}$ & $d f$ & $p$ & $\begin{array}{c}\text { Cohen's } \\
f^{2}\end{array}$ \\
\hline \multirow[t]{3}{*}{1} & IA & -0.07 & -0.18 & -1.6 & .113 & & & & & \\
\hline & $\begin{array}{l}\text { Brooding } \\
\text { Rumination }\end{array}$ & 0.85 & 0.4 & 3.81 & .000 & 0.20 & 9.96 & 2,79 & .000 & 0.25 \\
\hline & $\begin{array}{l}\text { IA } \mathrm{x} \\
\text { Brooding } \\
\text { Rumination }\end{array}$ & -0.03 & -0.21 & -1.92 & 0.058 & 0.24 & 3.69 & 1,78 & 0.058 & 0.05 \\
\hline
\end{tabular}

Note. Both Step 1 predictors are centered; $B=$ unstandardized regression coefficient; $p r=$ partial correlation between predictor and dependent measure; $R^{2}=$ Variance explained by the model (e.g., . $40=40 \%$ ); IA = Interoceptive Awareness 
Table 5. Linear Regression Model Predicting MASQ-S Anhedonic Depression.

\begin{tabular}{|c|c|c|c|c|c|c|c|c|c|c|}
\hline \multirow[b]{2}{*}{ Step } & \multirow[b]{2}{*}{ Predictor } & \multicolumn{4}{|c|}{ Regression Coefficients } & \multicolumn{5}{|c|}{ Regression Model } \\
\hline & & B & $p r$ & $t$ & $p$ & $R^{2}$ & $\begin{array}{c}F \\
\text { change }\end{array}$ & $d f$ & $p$ & $\begin{array}{c}\text { Cohen's } \\
f^{2} \\
\end{array}$ \\
\hline 1 & IA & -0.20 & -0.27 & -2.52 & .014 & & & & & \\
\hline & $\begin{array}{l}\text { Brooding } \\
\text { Rumination }\end{array}$ & 1.31 & 0.36 & 3.4 & .001 & 0.22 & 10.82 & 2,79 & .001 & 0.28 \\
\hline 2 & $\begin{array}{l}\text { IA } x \\
\text { Brooding } \\
\text { Rumination }\end{array}$ & -0.03 & -0.16 & -1.46 & 0.148 & 0.24 & 2.1 & 1,78 & .148 & 0.03 \\
\hline
\end{tabular}

Note. Both Step 1 predictors are centered; $B=$ unstandardized regression coefficient; $p r=$ partial correlation between predictor and dependent measure; $R^{2}=$ Variance explained by the model (e.g., . $40=40 \%$ ); IA = Interoceptive Awareness 
Figure 1. Decomposed Interaction of IA and Brooding Rumination on MASQ-S: General Distress

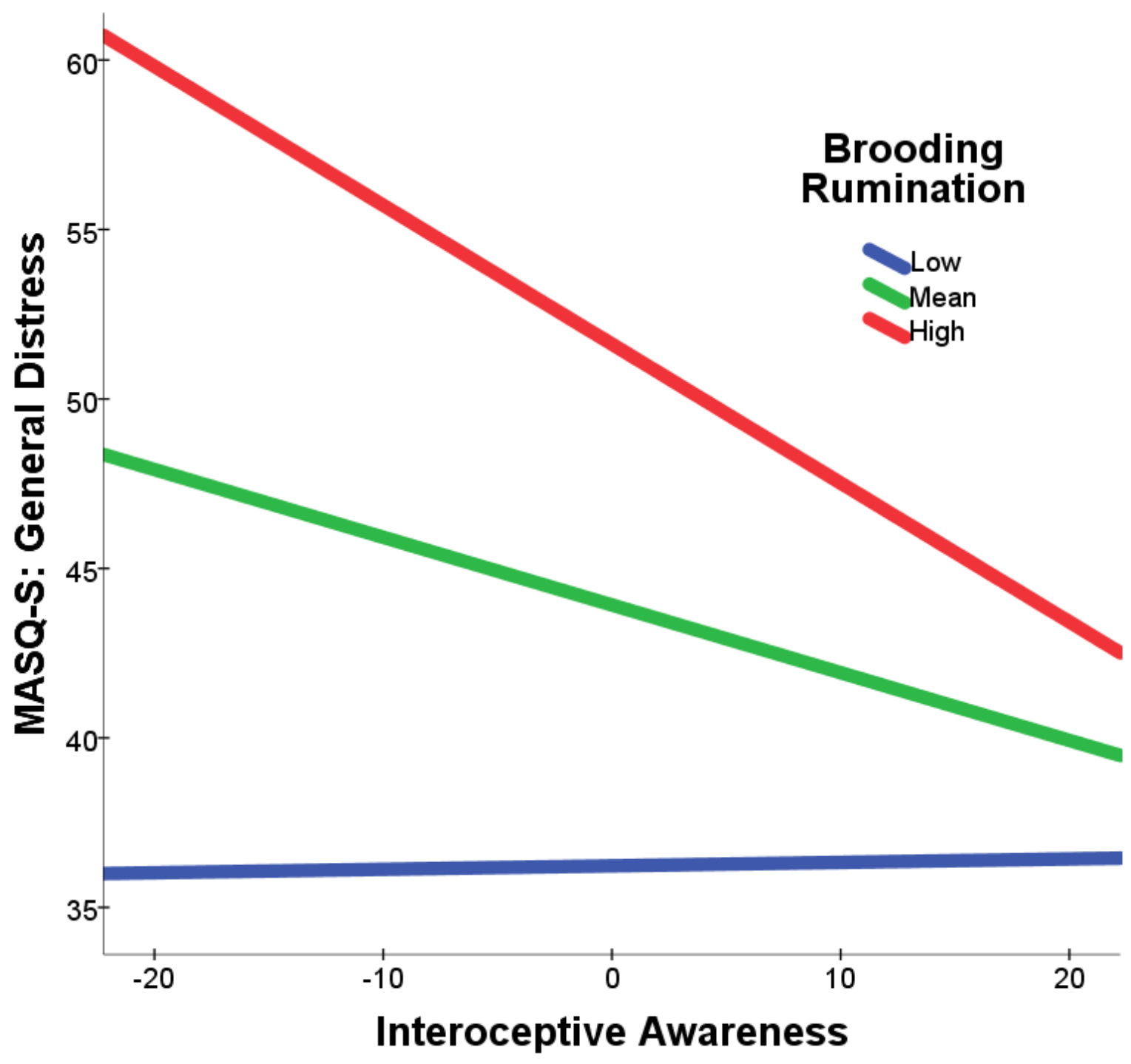




\section{References}

American Psychiatric Association. (2013). Diagnostic and Statistical Manual of Mental Disorders (DSM-5®). American Psychiatric Pub.

Armey, M. F., Fresco, D. M., Moore, M. T., Mennin, D. S., Turk, C. L., Heimberg, R. G., . . Alloy, L. B. (2009). Brooding and pondering: Isolating the active ingredients of depressive rumination with exploratory factor analysis and structural equation modeling. Assessment.

Barrett, L. F., Quigley, K. S., Bliss-Moreau, E., \& Aronson, K. R. (2004). Interoceptive sensitivity and self-reports of emotional experience. Journal of personality and social psychology, 87(5), 684 .

Beckwé, M., Deroost, N., Koster, E. H., De Lissnyder, E., \& De Raedt, R. (2014). Worrying and rumination are both associated with reduced cognitive control. Psychological research, 78(5), 651-660.

Berlucchi, G., \& Aglioti, S. M. (2010). The body in the brain revisited. Experimental brain research, 200(1), 25-35.

Borkovec, T. D., \& Inz, J. (1990). The nature of worry in generalized anxiety disorder: A predominance of thought activity. Behaviour Research and Therapy, 28(2), 153-158.

Borkovec, T. D., \& Ruscio, A. M. (2001). Psychotherapy for generalized anxiety disorder. Journal of Clinical Psychiatry, 62, 37-45.

Buckner, R. L., Andrews-Hanna, J. R., \& Schacter, D. L. (2008). The brain's default network. Annals of the New York Academy of Sciences, 1124(1), 1-38.

Butler, L. D., \& Nolen-Hoeksema, S. (1994). Gender differences in responses to depressed mood in a college sample. Sex Roles, 30(5-6), 331-346. 
Cameron, O. G. (2001). Interoception: the inside story—a model for psychosomatic processes. Psychosomatic medicine, 63(5), 697-710.

Casacalenda, N., Perry, J. C., \& Looper, K. (2002). Remission in major depressive disorder: a comparison of pharmacotherapy, psychotherapy, and control conditions. American Journal of Psychiatry.

Christoff, K., Gordon, A. M., Smallwood, J., Smith, R., Schooler, J. W., \& Posner, M. I. (2009). Experience Sampling during fMRI Reveals Default Network and Executive System Contributions to Mind Wandering. Proceedings of the National Academy of Sciences of the United States of America, 106(21), 8719-8724.

Craig, A. D. (2002). How do you feel? Interoception: the sense of the physiological condition of the body. Nature Reviews Neuroscience, 3(8), 655-666.

Craig, A. D. (2009). How do you feel—now? the anterior insula and human awareness. Nature Reviews Neuroscience, 10(1).

Craig, A. D. (2013). An interoceptive neuroanatomical perspective on feelings, energy, and effort. Behavioral and Brain Sciences, 36(06), 685-686.

Critchley, H. D., Wiens, S., Rotshtein, P., Öhman, A., \& Dolan, R. J. (2004). Neural systems supporting interoceptive awareness. Nature neuroscience, 7(2), 189-195.

Damasio, A. R. (1994). Descartes' error: Emotion, rationality and the human brain. New York: Putnam, 352.

Damasio, A. R. (1999). The Feeling of What Happens. Body and Emotion in the Making of Consciousness. Hartcourt. Inc. San Diego, New York. 
Davenport, P. W., Chan, P.-Y. S., Zhang, W., \& Chou, Y.-L. (2007). Detection threshold for inspiratory resistive loads and respiratory-related evoked potentials. Journal of applied physiology, 102(1), 276-285.

Davidson, R. J. (2004). Well-being and affective style: neural substrates and biobehavioural correlates. Philosophical Transactions-Royal Society of London Series B Biological Sciences, 1395-1412.

Domschke, K., Stevens, S., Pfleiderer, B., \& Gerlach, A. L. (2010). Interoceptive sensitivity in anxiety and anxiety disorders: An overview and integration of neurobiological findings. Clinical Psychology Review, 30(1), 1-11. doi: http://dx.doi.org/10.1016/j.cpr.2009.08.008

Dunn, B. D., Dalgleish, T., \& Lawrence, A. D. (2006). The somatic marker hypothesis: A critical evaluation. Neuroscience \& Biobehavioral Reviews, 30(2), 239-271.

Dunn, B. D., Dalgleish, T., Ogilvie, A. D., \& Lawrence, A. D. (2007). Heartbeat perception in depression. Behaviour Research and Therapy, 45(8), 1921-1930. doi: http://dx.doi.org/10.1016/j.brat.2006.09.008

Dunn, B. D., Evans, D., Makarova, D., White, J., \& Clark, L. (2012). Gut feelings and the reaction to perceived inequity: The interplay between bodily responses, regulation, and perception shapes the rejection of unfair offers on the ultimatum game. Cognitive, Affective, \& Behavioral Neuroscience, 12(3), 419-429.

Dunn, B. D., Galton, H. C., Morgan, R., Evans, D., Oliver, C., Meyer, M., . . Dalgleish, T. (2010a). Listening to Your Heart How Interoception Shapes Emotion Experience and Intuitive Decision Making. Psychological science, 21(12), 1835-1844.

Dunn, B. D., Stefanovitch, I., Evans, D., Oliver, C., Hawkins, A., \& Dalgleish, T. (2010b). Can you feel the beat? Interoceptive awareness is an interactive function of anxiety- and 
depression-specific symptom dimensions. Behaviour Research and Therapy, 48(11), 1133-1138. doi: http://dx.doi.org/10.1016/j.brat.2010.07.006

Ehlers, A. (1998). Why Van der Does, Van Dyck and Spinhoven (1997) did not demonstrate that superior heartbeat perception in panic disorder is partly an artefact. Journal of affective disorders, 49(3), 241-244.

Ehlers, A., \& Breuer, P. (1992). Increased cardiac awareness in panic disorder. Journal of Abnormal Psychology, 101(3), 371-382. doi: 10.1037/0021-843X.101.3.371

Eley, T. C., Stirling, L., Ehlers, A., Gregory, A. M., \& Clark, D. M. (2004). Heart-beat perception, panic/somatic symptoms and anxiety sensitivity in children. Behaviour Research and Therapy, 42(4), 439-448.

Farb, N. A., Anderson, A. K., Bloch, R. T., \& Segal, Z. V. (2011). Mood-linked responses in medial prefrontal cortex predict relapse in patients with recurrent unipolar depression. Biological psychiatry, 70(4), 366-372.

Farb, N. A. S., Daubenmier, J. J., Price, C. J., Gard, T., Kerr, C., Dunn, B., . . Mehling, W. E. (2015). Interoception, Contemplative Practice, and Health. Name: Frontiers in Psychology, 6, 763.

Farb, N. A. S., Segal, Z. V., \& Anderson, A. K. (2013). Mindfulness meditation training alters cortical representations of interoceptive attention. Social Cognitive and Affective Neuroscience, 8(1), 15-26. doi: 10.1093/scan/nss066

Farb, N. A. S., Segal, Z. V., Mayberg, H., Bean, J., McKeon, D., Fatima, Z., \& Anderson, A. K. (2007). Attending to the present: mindfulness meditation reveals distinct neural modes of self-reference. Social Cognitive and Affective Neuroscience, 2(4), 313-322. 
Ferguson, M. L., \& Katkin, E. S. (1996). Visceral perception, anhedonia, and emotion. Biological Psychology, 42(1), 131-145.

Furman, D. J., Waugh, C. E., Bhattacharjee, K., Thompson, R. J., \& Gotlib, I. H. (2013). Interoceptive awareness, positive affect, and decision making in major depressive disorder. Journal of affective disorders, 151(2), 780-785.

Füstös, J., Gramann, K., Herbert, B. M., \& Pollatos, O. (2012). On the embodiment of emotion regulation: interoceptive awareness facilitates reappraisal. Social Cognitive and Affective Neuroscience. doi: 10.1093/scan/nss089

Harshaw, C. (2015). Interoceptive dysfunction: Toward an integrated framework for understanding somatic and affective disturbance in depression. Psychological bulletin, 141(2), 311.

Herbert, B. M., Muth, E. R., Pollatos, O., \& Herbert, C. (2012). Interoception across Modalities: On the Relationship between Cardiac Awareness and the Sensitivity for Gastric Functions. PLoS ONE, 7(5), e36646. doi: 10.1371/journal.pone.0036646

Herbert, B. M., Pollatos, O., Flor, H., Enck, P., \& Schandry, R. (2010). Cardiac awareness and autonomic cardiac reactivity during emotional picture viewing and mental stress. Psychophysiology, 47(2), 342-354.

Herbert, B. M., Ulbrich, P., \& Schandry, R. (2007). Interoceptive sensitivity and physical effort: Implications for the self-control of physical load in everyday life. Psychophysiology, 44(2), 194-202.

James, W. (1884). II.-WHAT IS AN EMOTION? Mind(34), 188-205. 
Kerr, C. E., Sacchet, M. D., Lazar, S. W., Moore, C. I., \& Jones, S. R. (2013). Mindfulness starts with the body: somatosensory attention and top-down modulation of cortical alpha rhythms in mindfulness meditation. Frontiers in human neuroscience, 7.

Killingsworth, M. A., \& Gilbert, D. T. (2010). A wandering mind is an unhappy mind. Science, 330(6006), 932-932.

Kircanski, K., Thompson, R. J., Sorenson, J. E., Sherdell, L., \& Gotlib, I. H. (2015). Rumination and Worry in Daily Life Examining the Naturalistic Validity of Theoretical Constructs. Clinical Psychological Science, 2167702614566603.

Kirk, U., Downar, J., \& Montague, P. R. (2011). Interoception drives increased rational decisionmaking in meditators playing the ultimatum game. Frontiers in neuroscience, 5.

Knapp-Kline, K., \& Kline, J. P. (2005). Heart rate, heart rate variability, and heartbeat detection with the method of constant stimuli: slow and steady wins the race. Biological Psychology, 69(3), 387-396.

Knoll, J. F., \& Hodapp, V. (1992). A Comparison between Two Methods for Assessing Heartbeat Perception. Psychophysiology, 29(2), 218-222. doi: 10.1111/j.14698986.1992.tb01689.x

Mason, M. F., Norton, M. I., Van Horn, J. D., Wegner, D. M., Grafton, S. T., \& Macrae, C. N. (2007). Wandering minds: the default network and stimulus-independent thought. Science, 315(5810), 393-395.

McKiernan, K. A., D'Angelo, B. R., Kaufman, J. N., \& Binder, J. R. (2006). Interrupting the "stream of consciousness": an fMRI investigation. Neuroimage, 29(4), 1185-1191. 
Mehling, W. E., Price, C., Daubenmier, J. J., Acree, M., Bartmess, E., \& Stewart, A. (2012). The multidimensional assessment of interoceptive awareness (MAIA). PLoS ONE, 7(11), e48230.

Mennin, D. S., \& Fresco, D. M. (2013). What, Me Worry and Ruminate About DSM-5 and RDoC? The Importance of Targeting Negative Self-Referential Processing. Clinical Psychology: Science and Practice, 20(3), 258-267.

Montoya, P., Schandry, R., \& Müller, A. (1993). Heartbeat evoked potentials (HEP): topography and influence of cardiac awareness and focus of attention. Electroencephalography and Clinical Neurophysiology/Evoked Potentials Section, 88(3), 163-172.

Morris, J. S., Friston, K. J., Büchel, C., Frith, C. D., Young, A. W., Calder, A. J., \& Dolan, R. J. (1998). A neuromodulatory role for the human amygdala in processing emotional facial expressions. Brain, 121(1), 47-57.

Mussgay, L., Klinkenberg, N., \& Rüddel, H. (1999). Heart beat perception in patients with depressive, somatoform, and personality disorders. Journal of Psychophysiology, 13(1), 27-36.

Nolen-Hoeksema, S., \& Morrow, J. (1991). A prospective study of depression and posttraumatic stress symptoms after a natural disaster: the 1989 Loma Prieta Earthquake. Journal of personality and social psychology, 61(1), 115.

Nolen-Hoeksema, S., \& Morrow, J. (1993). Effects of rumination and distraction on naturally occurring depressed mood. Cognition \& Emotion, 7(6), 561-570.

Northoff, G. (2007). Psychopathology and pathophysiology of the self in depressionneuropsychiatric hypothesis. Journal of Affective Disorders, 104,1-14. doi:10.1016/j.jad .2007 .02 .012 
O'Brien, W. H., Reid, G. J., \& Jones, K. R. (1998). Differences in heartbeat awareness among males with higher and lower levels of systolic blood pressure. International journal of psychophysiology, 29(1), 53-63.

Olatunji, B.O., Naragon-Gainey, K., \& Wolitzky-Taylor,K.B. (2013). Specificity of rumination in anxiety and depression: A multimodal meta-analysis. Clinical Psychology: Science and Practice 20, 225-258.

Paulus, M. P. (2007). Decision-making dysfunctions in psychiatry-altered homeostatic processing? Science, 318(5850), 602-606.

Paulus, M. P., \& Stein, M. B. (2006). An insular view of anxiety. Biological psychiatry, 60(4), 383-387.

Paulus, M. P., \& Stein, M. B. (2010). Interoception in anxiety and depression. Brain structure and Function, 214(5-6), 451-463.

Phillips, M. L., Drevets, W. C., Rauch, S. L., \& Lane, R. (2003). Neurobiology of emotion perception I: The neural basis of normal emotion perception. Biological psychiatry, 54(5), 504-514.

Pineles, S. L., \& Mineka, S. (2005). Attentional biases to internal and external sources of potential threat in social anxiety. Journal of Abnormal Psychology, 114(2), 314.

Pizzagalli, D. A., Iosifescu, D., Hallett, L. A., Ratner, K. G., \& Fava, M. (2008). Reduced hedonic capacity in major depressive disorder: evidence from a probabilistic reward task. Journal of psychiatric research, 43(1), 76-87.

Pollatos, O., Kirsch, W., \& Schandry, R. (2005). On the relationship between interoceptive awareness, emotional experience, and brain processes. Cognitive Brain Research, 25(3), 948-962. 
Pollatos, O., \& Schandry, R. (2008). Emotional processing and emotional memory are modulated by interoceptive awareness. Cognition \& Emotion,22(2), 272-287.

Pollatos, O., Schandry, R., Auer, D. P., \& Kaufmann, C. (2007). Brain structures mediating cardiovascular arousal and interoceptive awareness. Brain Research, 1141(0), 178-187. doi: http://dx.doi.org/10.1016/j.brainres.2007.01.026

Pollatos, O., Traut-Mattausch, E., \& Schandry, R. (2009). Differential effects of anxiety and depression on interoceptive accuracy. Depression and anxiety, 26(2), 167-173.

Prinz, J. J. (2004). Gut reactions: A perceptual theory of emotion: Oxford University Press.

Raichle, M. E., MacLeod, A. M., Snyder, A. Z., Powers, W. J., Gusnard, D. A., \& Shulman, G. L. (2001). A default mode of brain function. Proceedings of the National Academy of Sciences of the USA, 98, 676-682. doi: 10. 1073/pnas.98.2.676

Reiss, S., Peterson, R. A., Gursky, D. M., \& McNally, R. J. (1986). Anxiety sensitivity, anxiety frequency and the prediction of fearfulness. Behaviour research and therapy, 24(1), 1-8.

Ring, C., Brener, J., Knapp, K., \& Mailloux, J. (2015). Effects of heartbeat feedback on beliefs about heart rate and heartbeat counting: A cautionary tale about interoceptive awareness. Biological Psychology, 104, 193-198.

Sanislow, C. A., Pine, D. S., Quinn, K. J., Kozak, M. J., Garvey, M. A., Heinssen, R. K., . . . Cuthbert, B. N. (2010). Developing constructs for psychopathology research: research domain criteria. Journal of Abnormal Psychology, 119(4), 631.

Schachter, S., \& Singer, J. (1962). Cognitive, social, and physiological determinants of emotional state. Psychological review, 69(5), 379.

Schandry, R. (1981). Heart Beat Perception and Emotional Experience. Psychophysiology, 18(4), 483-488. doi: 10.1111/j.1469-8986.1981.tb02486.x 
Schandry, R., Bestler, M., \& Montoya, P. (1993). On the relation between cardiodynamics and heartbeat perception. Psychophysiology, 30(5), 467-474. doi: 10.1111/j.14698986.1993.tb02070.x

Sze, J. A., Gyurak, A., Yuan, J. W., \& Levenson, R. W. (2010). Coherence between emotional experience and physiology: does body awareness training have an impact? Emotion, 10(6), 803.

Tang, Y.-Y., Hölzel, B. K., \& Posner, M. I. (2015). The neuroscience of mindfulness meditation. Nature Reviews Neuroscience, 16(4), 213-225.

Teasdale, J. D. (1999). Emotional processing, three modes of mind and the prevention of relapse in depression. Behaviour research and therapy, 37, S53-S77.

Treynor, W., Gonzalez, R., \& Nolen-Hoeksema, S. (2003). Rumination reconsidered: A psychometric analysis. Cognitive Therapy and Research, 27(3), 247-259.

Watkins, E., \& Moulds, M. (2005). Distinct modes of ruminative self-focus: impact of abstract versus concrete rumination on problem solving in depression. Emotion, 5(3), 319.

Watkins, E., \& Teasdale, J. D. (2004). Adaptive and maladaptive self-focus in depression Journal of affective disorders, 82(1), 1-8.

Watson, D., \& Clark, L. A. (1991). The mood and anxiety symptom questionnaire (MASQ). Unpublished manuscript, University of Iowa, Iowa City

Watson, D., Clark, L. A., Weber, K., Assenheimer, J. S., Strauss, M. E., \& McCormick, R. A. (1995a). Testing a tripartite model: II. Exploring the symptom structure of anxiety and depression in student, adult, and patient samples. Journal of Abnormal Psychology, 104(1), 15. 
Watson, D., Weber, K., Assenheimer, J. S., Clark, L. A., Strauss, M. E., \& McCormick, R. A. (1995b). Testing a tripartite model: I. Evaluating the convergent and discriminant validity of anxiety and depression symptom scales. Journal of Abnormal Psychology, 104(1), 3.

Webster, K. E., \& Colrain, I. M. (2000). The relationship between respiratory-related evoked potentials and the perception of inspiratory resistive loads. Psychophysiology, 37(06), 831-841.

Werner, N. S., Jung, K., Duschek, S., \& Schandry, R. (2009). Enhanced cardiac perception is associated with benefits in decision-making.Psychophysiology, 46(6), 1123-1129.

Werner, N. S., Mannhart, T., Reyes Del Paso, G. A., \& Duschek, S. (2014). Attention interference for emotional stimuli in cardiac interoceptive awareness. Psychophysiology, 51(6), 573-578.

Werner, N. S., Peres, I., Duschek, S., \& Schandry, R. (2010). Implicit memory for emotional words is modulated by cardiac perception. Biological psychology, 85(3), 370-376.

Whitehead, W. E., Drescher, V. M., Heiman, P., \& Blackwell, B. (1977). Relation of heart rate control to heartbeat perception. Biofeedback and Self-regulation, 2(4), 371-392.

Wiens, S. (2005). Interoception in emotional experience. Current opinion in neurology, 18(4), $442-447$.

Wiens, S., Mezzacappa, E. S., \& Katkin, E. S. (2000). Heartbeat detection and the experience of emotions. Cognition \& Emotion, 14(3), 417-427.

Yates, A. J., Jones, K. E., Marie, G. V., \& Hogben, J. H. (1985). Detection of the heartbeat and events in the cardiac cycle. Psychophysiology, 22(5), 561-567. 
Yoris, A., Esteves, S., Couto, B., Melloni, M., Kichic, R., Cetkovich, M., . . Ibanez, A. (2015). The roles of interoceptive sensitivity and metacognitive interoception in panic. Behavioral and Brain Functions, 11(1), 14.

Zechman, F., Hall, F. G., \& Hull, W. E. (1957). Effects of graded resistance to tracheal air flow in man. Journal of applied physiology, 10(3), 356-362.

Zhao, W., Martin, A. D., \& Davenport, P. W. (2002). Detection of inspiratory resistive loads in double-lung transplant recipients. Journal of applied physiology, 93(5), 1779-1785. 